\title{
Characteristic of materials for the 3D printed building constructions by additive printing
}

\author{
Katarzyna Pacewicz ${ }^{1, *}$, Anna Sobotka ${ }^{2}$, and Lukasz Gołek ${ }^{1}$ \\ ${ }^{1,2.3}$ AGH University of Science and Technology, al. Mickiewicza 30, 30-059 Cracow, Poland
}

\begin{abstract}
Three dimensional printing is a promising new technology to erect construction objects. Around the world in every moment a new prototypes constructions are made by using this method. Three dimensional printing is taken into account as technology which can be used to print constructions in automated way on the Moon or Mars. The raw materials, which can be used with three dimensional printing have to fulfil basic requirements for those which are used in construction. That means that components of printing mortars are made from ingredients easily accessible in area nearby construction site and can be reusable. The cost of printing building objects due to that requirements is comparable to costs of traditional building, which are currently available. However additive techniques of printing needs a dedicated mortars for printer supplying. Characteristic for such mortars is: setting time, compressive strength, followability in the printing system, shape stability of every printed layer, controlling the hydration rate to ensure bonding with the subsequent layer, reusable capabilities, easily accessible raw materials, cost of such mixtures shouldn't be too high in order to keep 3D printing competitive for traditional ways of building, mortar components should be recyclable and printing process should not influence negatively on an environment and people. All properties of printing mortars are determined by the device for additive application method. In this paper review of available materials used for three dimensional printing technology at construction site is presented. Presented materials were analysed in terms of requirements for building materials technology. Due to the lack of detailed information's in available literature, regarding to the properties of raw materials, the results of this analysis may be used in the designing of new concrete mixtures for the use in three-dimensional printing technology for construction.
\end{abstract}

\section{Introduction}

Three dimension printing is a process of making three-dimensional, physical objects based on computer model. At the beginning this technology was used to make device's prototypes and machine's elements. The development of three-dimensional printing technology accelerate the process of designer's vision became true.

Commercialized and widely admitted the 3-dimensional printing technology is stereolithography, which was patented in 1986 by Charls Hull. His method uses local

*Corresponding author: kwronska@agh.edu.pl 
hardening photopolymer resins by a strong laser light beam. In 1989 Carl Deckard patented Selected Laser Sintering (SLS), which also basis on hardening material by laser beam. This solution is very similar to stereolithography, however powder supplements $s$ needs to be used. The most popular 3-dimensional printing technology is Fused Deposition Modeling (FDM), which was patented in 1992. In FDM method the thermoplastic material is extruded by heated by printer's nozzle. The nozzle and temperature controls the melted material flow. Object is created layer by layer. Firstly the object contour is drawn, than the created area needs to be filled up. In order to solve issue with elements which needs supports the additional printhead is used. One printhead provides building material, while the second provides material for support, which can be water-soluble or separated from printed element. FDM uses thermoplastic materials, with high mechanical strength, thermal resistance and chemical durability. 3 Dimensional printing (3DP) puts thin layer of powdered composite, which is blended with the sprayed binder, from the printer's head moved in horizontal plane. When the layer is finished, roller puts new layer of powder material on lowered machine's table. In this technology the additional supports are not required. It allows to build complex structures, if the object thickness and its strength allows to safely remove it from working chamber. Objects made with 3DP method are fragile and damage sensitive. In order to increase their mechanical strength, appropriate treatment is performed and then hardener and UV protection agents should be applied. The 3DP is currently available in three different variants, where as supplements the ceramic, plastic and powdered metal materials are used [12].

Currently a lot of constructions around the world are built with 3-dimensional printing technology and China is leading this industry branch, however there is a lack of information about used formulas and mixtures, which could be used for printing technology. Therefore, the authors, based on the analysis of the available literature and the analysis of the concrete mixtures, attempted to combine the required characteristics of materials and mortars that provide the ability to use it as supply materials for printers and meet the key requirements such as the compressive and bending strength. This article contains the description of chosen implementations and materials used for it, such as raw materials and mortar mixtures. The influence of mortar components on the properties of fresh and hardened mortar were analysed. Results can be used to design new concrete recipes for construction printing.

\section{Three dimensional printing process in construction}

The print process of the ready element consists of seven stages. Stage one is the graphical 3D model computer designing (mostly CAD). Next step is conversion of prepared model into format, which is understandable by 3D printer. Usually this is STL format. Before printing, the printer is adapted to work and the drawing file is adapted to set the print scale and orientation of printed items. Following stage is $3 \mathrm{D}$ object printing. Material is laid layer by layer with specified thickness, according to 3D model. When the printing process is finished, printer needs to be clean up. Last stage is object's finishing.

Automation of building processes can result in a reduction of the work force, which increases safety at the construction site, reduces construction time and the production costs. In addition, the development of building information modelling and the use of digital information streamlines the automation and production process in construction. Currently, there are several projects related to creation of constructions in the additive technology, namely: Apis-Cor, Contour Crafting, D-Shape, Concrete Printing.

Russian's company Apis-cor [1] has printer capable to work at construction site. Printer's size is $4 \mathrm{~m} / 1.6 \mathrm{~m} / 1.5 \mathrm{~m}$ and weighs 2 tons (figure 1). The printer is placed on one base (foot). A special support system does not require the terrain preparation. Device work 
like construction crane, that allows printer to rotate and print object's inside and outside. Printer's work space is $132 \mathrm{~m}^{2}$. Mixture for printing is based on cement.

Final hardened material from such recipe indicate the properties like the standard $\mathrm{C} 16 / 20$ concrete. In this project the geopolymers and fiber concrete are also used. The foundation is firstly created by drawing the contour, next it's reinforced and filled up with concrete. After the foundation and insulation works, the contours of the walls are printed and reinforced with the zigzag shape inside the wall.

Cooperation with Geobeton company brings a new recipes for concrete to use in construction industry. Geocement ${ }^{\mathrm{TM}}$ consists of two components: powder geopolymer cement and the liquid reagent - geosilicate. Additionally, the company offers dry geopolymer concrete, which is delivered at construction side in custom amounts for concrete production. Geopolymer structure and high level of mesoporosity brings the air flow through material, so it creates insulation, frost and fire resistance. Some other properties are very adjustable, like thixotropy or slump. The setting time in very wide range, up to 180 minutes. Geopolymer concretes indicate high compressive strength, over $100 \mathrm{MPa}$ and chemical resistance on alkalis and acids. On the basis of a geopolymer binder, various compositions of geo-concrete were developed and tested. The tests were carried out in Irkutsk, Russia. The industrial by products were used in the developed recipe [1].

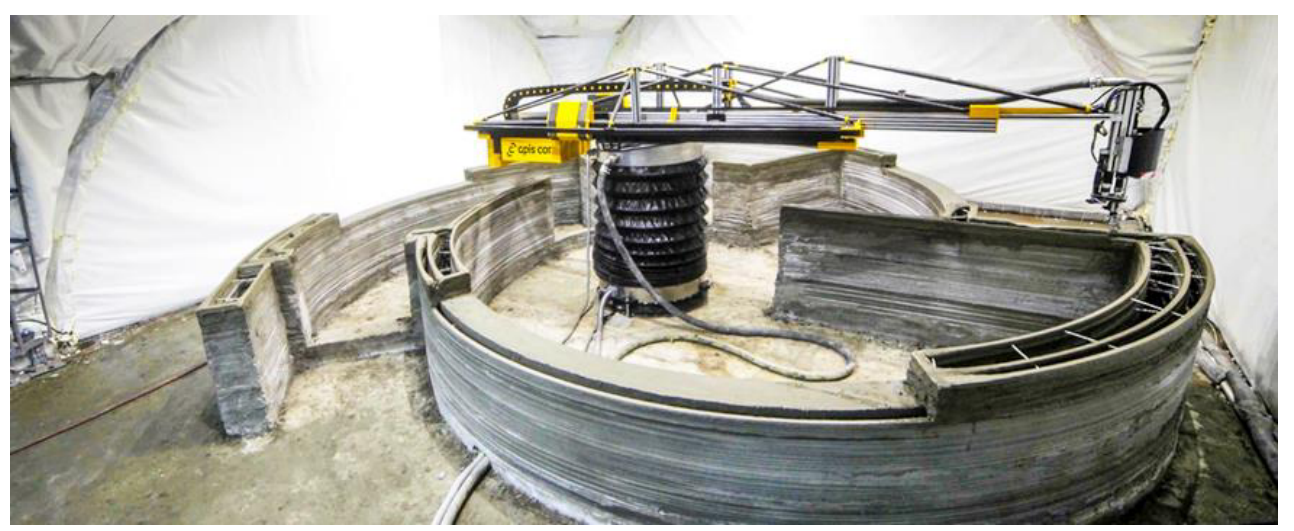

Fig. 1. Printing process presented in February 2017 in Stupino city with Apis Cor construction 3D printer [1]

The Contour Crafting project was developed by the team of professor Behrokh Kloshnevisfrom the University of Southern California. It is a device mounted to the crane frame. The robot's additional arm installs appropriate channels to supply the facility with media and horizontal structural elements, which are difficult to print. This 3D printing technology involves the additive application of cement mortar with a trowel-tipped head. The trowel usage allows to smooth the unevenness of the side surfaces, which are result of layers addition. Currently, the head is capable of additive construction of a full-sized structural wall, with the minimal consumption of material $[6,12]$.

The D-Shape printer can print monolithic structures (figure 2). It uses a powder deposition process, which is selectively cured by means of a binder. In this method, the printing process consists in arranging the appropriate thickness of the building material, compaction of the layer and application of the binder by the nozzle mounted on the ramp. After the finished printing process, the printed object is extracted from the loose powder bed. The dry material, which was not treated by the binder is used as a support for the constructed objects, additionally it can be used in the further printing process $[5,6]$.

Cementitious Micro-Concrete printing process uses materials with various granulation, from $0.2 \mathrm{~mm}$ to $5 \mathrm{~mm}$. Material binding can be carried out with water solutions or cement binder. The inert raw material may be: crushed stone, sand, gravel, crushed clay aggregates 
and ceramics. Also recycled materials can be used, such as: glass, wood chips, shredded plastics, demolition materials, rubber, gypsum or a mixture of the above mentioned batches, reinforced by fibers: natural, glass, basalt, polymer or steel. As a liquid binders and reagents for inert batches are used: suspensions based on Portland cement, Sorel cement or geopolymers, polymeric primers (urethane, silossane, vinyl with the exposition of fenolic and $\mathrm{PBB}, \mathrm{PPC}$ binder) all with $\mathrm{v} \leq 3^{\circ} \mathrm{E}[2]$.

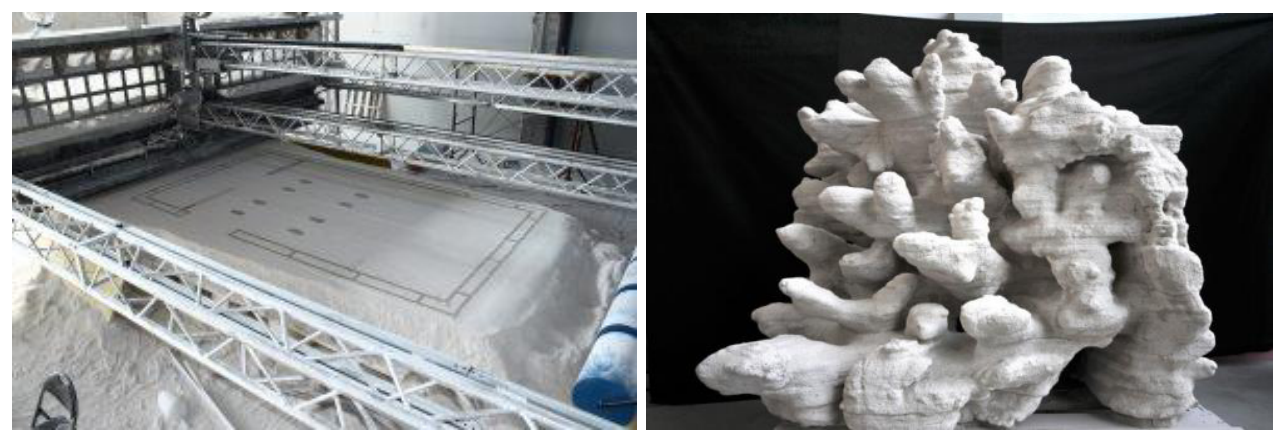

Fig. 2. D-Shape printer (on the left) and printed object - coral reef (on the right) [2]

Examinations on "Concrete Printing" project are carried out by Loughborough University $[4,5,6,7]$. Printing device prototype is made from steel frame with dimensions $5.4 \mathrm{~m}$ length, $4.4 \mathrm{~m}$ wide, $5.4 \mathrm{~m}$ height and printing head is mounted to moveable horizontal bar (figure 3). Printing process can be described in three steps: data preparation concrete mixture preparation and printing. Prepared mixture is placed in the pump on the external platform, in order to feed it through the printing nozzle with diameter $9 \mathrm{~mm}$. The proposed solution provides small buffer tank, placed on the top of printer.

This project uses two kinds of materials for printing. The cement-based material is used to build the elements, while the gypsum-based material serves as support material for the printed element. Gypsum material was used in this project due to easy material removal, low mechanical strength and $100 \%$ recycling. Retard admixtures are added as a mixture components to provide required open time and to obtain a constant workability of the material during production.
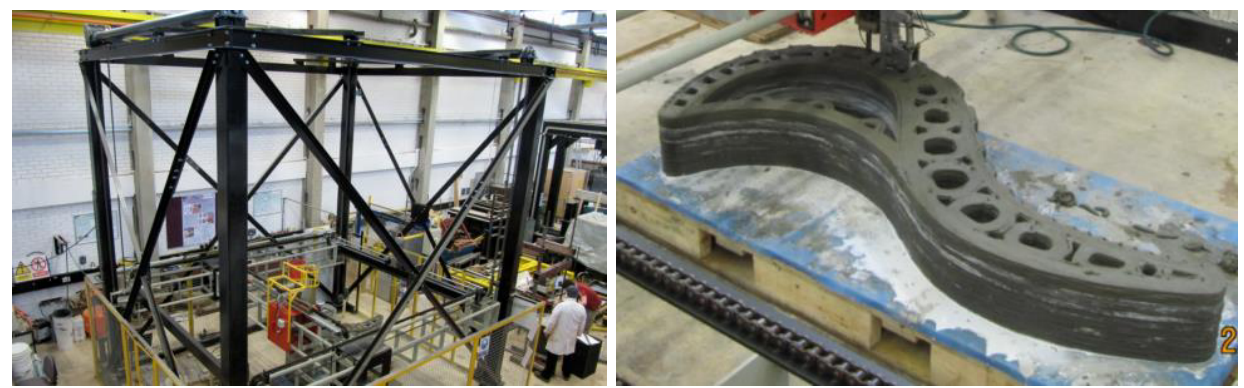

Fig. 3. Concrete printing system (on the left) and printed bench (on the right) [4]

The research described in paper [4] uses sand with a maximum grain size of $2 \mathrm{~mm}$. As a binding components the cement CEM I 52,5, fly ash and non-compressed silica dust were used. The dry components were added to mixing water together with polycarboxylate-based superplasticizer to increase the workability and material strength. In order to obtain the proper open time, the retarder based on amino-tris (methylene phosphonic acid), citric acid and formaldehyde was added. Studies were also carried out using an accelerator based on sulfones, aluminum salts and diethanolamine. The concrete also contained polypropylene microfibers with a length of $12 \mathrm{~mm}$ and a diameter of $0.18 \mathrm{~mm}$, which were designed to reduce shrinkage and deformation in the plastic state. 


\section{Characteristic of mixture's components for additive printing}

Presented examples of 3D printing projects of buildings and used materials are not described in details, but allows to analyse the required features of a mortar mixtures that can be used in 3D printers for printing of building objects. For 3D printing the research teams, mentioned in the article, mostly used cement-based materials. Of course the material used for 3D printing has to be compatible with 3D printer. In the composition of modern concrete, additives and admixtures are present along with aggregates, cement and water, which are added to improve selected properties of mortars.

Additives can significantly modify properties of fresh or hardened mortar. While designing mixtures of mortars useful for 3D printing, the care should be taken that the mortar meets the performance assumptions of fresh and hardened mortars. When designing the composition of mortar, to the necessity of extrusion should be taken into account, what is influencing on workability and open time. The key properties of hardened concrete are compressive and bending strength.

Additives used to produce concrete include: ground granulated blast-furnance slag, fly ash, silica fume, and rock powders: limestone and quartz powders, which are treated as chemically passive components.

Fly ash - its wide application is determined by its high fineness, chemical and phase composition as well as pozzolanic activity. Fly ash improves workability of mortar mixtures and significantly slows down mortars hardening process. Concrete shrinkage decreases in proportion to amount of cement replaced by fly ash. Fly ashes with high content of unburned coal lowers the effectivity of chemical admixtures, especially aeration, plasticizers of superplasticizers. Silicoeous fly ashes improves mixtures workability, that improves its pumping process.

Ground granulated blast furnace slag is obtained by rapid cooling of liquid slag, which is formed as a by-product in the production of a pig iron in a blast furnace. Then it is milled, with the agents facilitating its grinding which should not exceed $1.0 \%$. The organic components should not exceed $0.2 \%$ of the slag mass. Slag is more active and more effectively seals concrete in relation to fly ash.

Silica fume is a by-product of the production of metallic silicon or its alloys. Silica dust seals the microstructure of concrete by filling the space between cement grains. The use of silica dust in an amount of $2-3 \%$ (as an admixture) of cement mass, has a beneficial effect on improving workability of the mixture along with good viscosity. Silica dust forms a sol with water, what prevent water outflowing of the mixture while compacting. Rock powders improve the workability of mortars. The most beneficial effects are obtained by using powders obtained from low-crystalline limestone rocks. Bentonite, as a clay rock, improves the tixotropic properties of mortar mixtures. This material contains mainly of aluminosilicates, which influenced by water swell and increase their volume several times.

Synthetic resins show a significant adhesion with aggregate and hardened mortar. A properly selected composition of resin improves workability of the mortar as well as mechanical strength and physical properties $[3,8]$.

The role of admixtures is to improve one or several properties of a mortars or hardened concrete. They are added in amount up to $5 \%$ of the cement mass, during mixing. While designing mortars for $3 \mathrm{D}$ printing, it is important to focus on effects of modifications caused by admixtures, which can improve one and worsen the another. Superplasticizers are based on the synthetic resins that give a negative charge to the cement grains, which facilitates the formation of a water layer between the cement grains. Due to the interaction of electrostatic forces between the molecules, the workability of mixture is improved. Mixture internal friction and surface tension of the water are reduced. The process of wetting the aggregate and binder is easier, and the mortar mixtures becomes more plastic. 
The selection of the right superplasticizer depends on the cement properties and also on additives and on aggregates (to some extent). Difficulty in selection results from the differences between the binder and the superplasticizer properties.

Admixtures that accelerate hardening do not cause violent binding reaction, only shorten it, which accelerates the hardening period. These admixtures are designed to increase the dynamics of mortar strength increase over time. Process of initial strength increase is accelerated, with no negative effects on the final mortar strength. While printing successive layers of the object, the initial mortar strength is very important to prevent delamination and printed structure disintegration.

Retarding admixtures are used because during the process of printing the transport of the mixture takes a long time and it is needed to obtain proper bonding between particular mortar layers. Retarding of the bond causes the transported mixture to retain its liquidity for longer time, which has a positive effect on the pumping and printing processes. Concrete with a retardant admixture is indicate lower initial strength and usually slower hardening.

The viscosity modifying admixtures are designed to increase the viscosity of mortars, without causing significant changes in consistency. They are usually used to eliminate segregation, especially in self-compacting mixtures. Consideration should be given to the technology of feeding the mixture to the $3 \mathrm{D}$ printer, because while pumping the mixture, increasing the viscosity requires a higher pumping pressure $[3,8]$

\section{Additive printing mixes conditions and specific features}

Based on previous research and experiences of 3D printing technology in construction, especially for building structures, it is possible to determine the principles the composition of mortar mixtures and its required properties (Fig. 4). It should be taken into account many different implementations, conditions and variants of buildings, in particular the specific conditions of the construction site. While designing a mortar mixture intended for use is $3 \mathrm{D}$ printing technology, it should be noted that this mixture meets the accepted criteria and design assumptions.

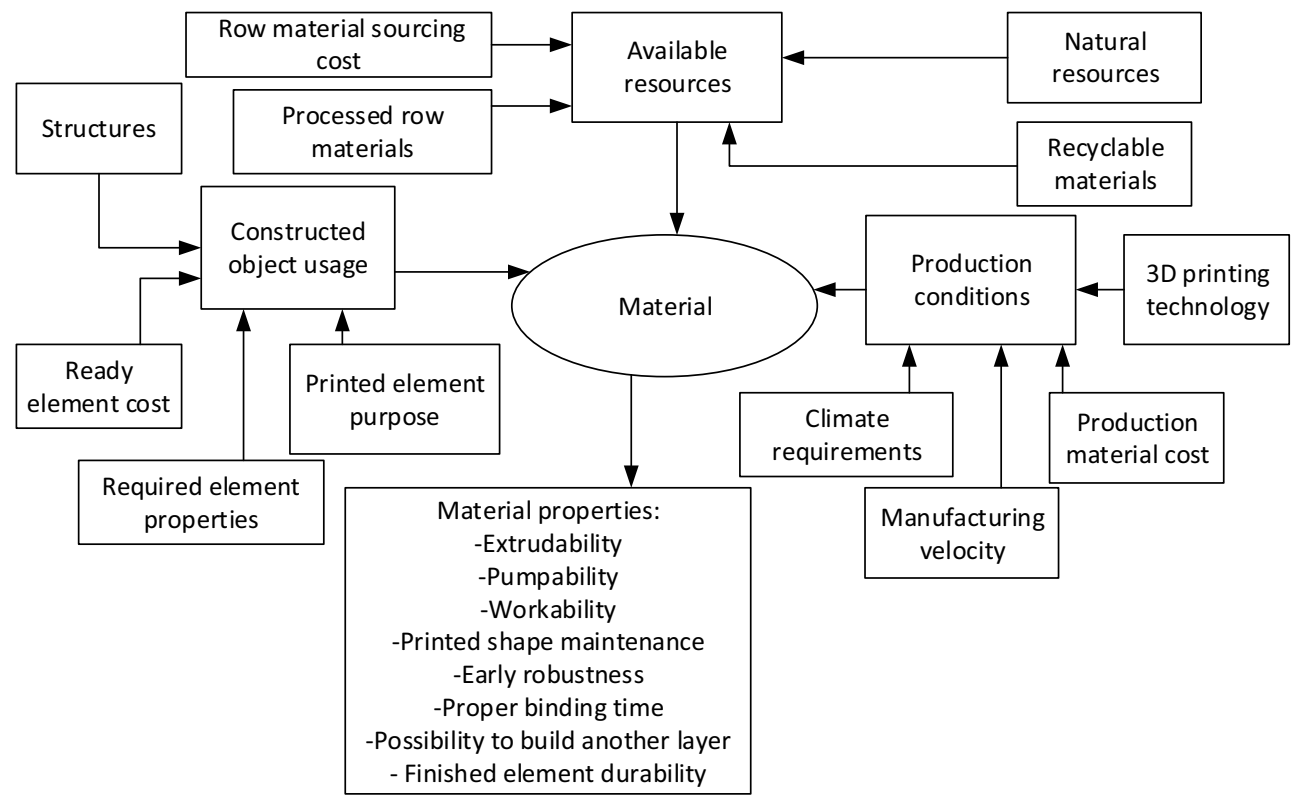

Fig. 4. Building material composition for $3 \mathrm{D}$ printing and properties requirements 
Material, designed and used in 3D printing technology for construction, depends on three interpenetrating factors: raw materials, methods of application and methods of production. While designing such a material, it is also necessary to pay attention to the properties that the manufactured object has to fulfil and that it is possible to print by the given printer. Designer should pay attention to the locally available raw materials, near the construction site, so that the cost of their transport would make economic sense. The composition of the mixture is also influenced by climatic conditions at the place of object's production, as it affect its composition and maturing conditions. The time after which the given properties will be achieved also affects the printing process, it also depends on the method of material delivery and the time of applying subsequent layers of building material. The composition of the material also depends on the cost of producing a given object.

As stated above, while designing and performing 3D printing from a mortar mixtures, it should pay attention to its properties that affect the possibility and quality of the printed object. Particular attention should be paid to such parameters as: flowability of the mixture, extrudability, stability of the printed shape, bonding time, strength and shrinkage of concrete.

The flowability of the mixture allows the pumping the mixture along the feeding and mixing systems. Regulation of the mixture rheology and its flow in the fresh state is ensured due to the wide particle size distribution. The use of different granulation of additives contributes to a denser packing of the mix grains, which results in better fluidity. It should be noted that too much dusty fraction (increase of intermolecular friction) can increase the viscosity of the mixture, which will negatively affect its fluidity.

Extrudability is the ability of the mixture to be transported over the small tubes and nozzles in the print head. The workability and consistency of the mixture have a fundamental influence on the extrudability. In order to obtain a good extrudability of the mixture, concrete should be designed in accordance to the principles of designing selfcompacting concrete and spraying concrete.

The ability to preserve printed shape is another important parameter that should be met by the pumped mixture used for 3D printing. The printed layers of material should keep their shape formed by nozzles under the pressure of subsequent layers applied by the printer. The decisive parameter is the early strength of the hardened mixture. The shape stability is ensured by increased amount of fine aggregate and sand. Viscosity modifying admixtures can reduce the overflowing of the mixture and improve the stability of the printed structure.

The setting time affects the possibility of mixing during printing. Workability, which affects possibility of extrusion, needs a long "opening time". Otherwise, the material, becomes stiffer and causes the slower flow of the mixture through the feed systems of the printer, or blocks the printer. Additionally, the long open time helps in connecting material between the layers, but it can negatively affect the formation of printed elements. Short bonding time is needed while laying successive layers of material, to keep the shape and load of the higher applied layers. The strength and stiffness of the printed shape is obtained due to the increased density of the matrix obtained by using of finer powders than Portland cement. The strength of the mixture is also influenced by: water-cement ratio, cement strength class, aggregate quality and the presence of admixtures and additives. The contraction of the concrete may start already in the binding period as well as in the initial hardening period. Shrinkage significantly affects the dimensional accuracy and stability of printed objects. Shrinkages are caused by evaporation of water from external layers and changes of the temperature increase caused by heat of hydration. Printed elements have a much larger surface exposed to the influence of ambient conditions in relation to the elements made with the formwork. While designing, it should be noted that the shrinkage 
increases, among others, with the increase the amount of water in the past, cement fineness and grain size of aggregate.

\section{Summary}

3D printing technology development in the industry has contributed to the large technological jump. Thanks to various methods of object production, 3D printing enables testing on prototypes, previously difficult to perform with normal production methods.

This paper focuses on 3D printing technology currently used in construction. 3D printing of mortar mixtures is a promising method that can revolutionize the traditional building processes. Safety for building is increased through highly effective automation of the building process, and the demand for workforce is reduced. The costs of creating of new construction objects can be much cheaper compared to traditional building methods. The basic issue in $3 \mathrm{D}$ printing of buildings is, except the printer, the material for printing. Many new materials have already been developed, among others: biological origined, steel, wooden and plastics, allowing additive printing of products. However, it seems that for each project designed for implementation in additive printing technology, an individual composition of the printing mixture should be designed.

The designed mixture for printing the object first of all should have certain specific features that enable printing and standard requirements that must pass the particular elements of the building. Due to that whole building could meet the required standards, like strength, fire protection, etc. On the other hand, the raw materials for the production of mixtures should be selected in accordance with the concept of sustainable development, ie saving non-renewable resources, waste materials that can be recycled, including possible minimization of costs. Modelling the mixture properties of, selection of its properties also results from the function that printed construction or the element in the printed object will perform. Also, implementation conditions, including climatic conditions, may affect the required characteristics of the used mixture.

Mixtures for printing should have an advantageous printable properties that can be controlled in the wide range and modify their properties to improve performance during the $3 \mathrm{D}$ printing process. Preparing a compatible material for a large-scale 3D printer is a difficult task. There are various factors, which affect on the properties of fresh and hardened mortars. The detailed analysis of the properties and consideration of the mechanisms is definitely recommended due to action of individual additives and admixtures affect on the workability of the printing mixtures. Also due to the capabilities of the printer. Although this technology is in experimental phase, 3D printing from mortar mixtures can revolutionize and increase the potential of the construction sector.

\section{References}

1. Apis cor, w:http://apis-cor.com available 15.04.2018.

2. D-Shape, w:https://d-shape.com available 15.04.2018.

3. Z. Jamroży, Beton i jego technologie, PWN, Warszawa (2015)

4. T.T. Le, S. A. Austin, S. Lim, R. A. Buswell, A. G. F. Gibb, T. Thorpe, Mix design and fresh properties for high-performance printing concrete, Materials and Structures, $\mathbf{4 5}$, 1221-1232, (2012) 
5. T. T. Le, S. A. Austin, S. Lim, R. A. Buswell, R. Law, A. G. F. Gibb, T. Thorpe, Hardened properties of high-performance printing concrete, Cement and Concrete Research, 42(3), 558 - 566, (2012)

6. S. Lim, R. A. Buswell, T. T. Le, S. A. Austin, A. G. F. Gibb, T. Thorpe, Developments in construction-scale additive manufacturing processes, Automation in Construction, 21(1), 262-268, (2012)

7. S. Lim, T. Le, J. Webster, R. Buswell, S. Austin, A. Gibb, T. Thorpe Fabricating construction components using layered manufacturing technology, (GICC'09), Loughborough University, pp. 512-520, (2009)

8. G. Ma, L. Wang, A critical review of preparation design and workability measurement of concrete material for largescale $3 D$ printing, Frontiers of Structural and Civil Engineering, 1-19, (2017)

9. Z. Malaeb, H. Hachem, A. Tourbah, T. Maalouf, N. El Zarwi, F. Hamzeh, 3D Concrete Printing: Machine and Mix Design, IJCIET, 6(6), 14-22, (2015)

10. B. Nematollahi, M. Xia, J. Sanjayan, Current Progress of $3 D$ Concrete Printing Technologies, ISARC 2017, 260-267, (2017)

11. V. N. Nerella, M. Krause, M. Näther, V. Mechtcherine, Studying printability of fresh concrete for formwork free Concrete on-site 3D Printing technology (CONPrint3D), $25^{\text {th }}$ Conference on Rheology of Building Materials, Regensburg, 236 - 246, (2016)

12. A. Sobotka, K. Wrońska, 3D w budownictwie, Cz.1,2 —Capabilities of use the threedimensional printing technology in construction industry, Builder, 11,12, pp 26-29, (2015) 